\title{
Recognition of Planar Segments in Point Cloud based on Wavelet Transform
}

\author{
Zivana Jakovljevic, Radovan Puzovic, and Miroslav Pajic, Member, IEEE
}

\begin{abstract}
Within industrial automation systems, 3D (three dimensional) vision provides very useful feedback information in autonomous operation of various manufacturing equipment (e.g., industrial robots, material handling devices, assembly systems, machine tools). The hardware performance in contemporary 3D scanning devices is suitable for on-line utilization. However, the bottleneck is the lack of real-time algorithms for recognition of geometric primitives (e.g., planes, natural quadrics) from a scanned point cloud. One of the most important and the most frequent geometric primitive in various engineering tasks is plane. In this paper, we propose a new, fast, one-pass algorithm for recognition (segmentation and fitting) of planar segments from a point cloud. To effectively segment planar regions, we exploit the orthonormality of certain wavelets to polynomial function, as well as their sensitivity to abrupt changes. After segmentation of planar regions, we estimate the parameters of corresponding planes by using standard fitting procedures. For point cloud structuring a z-buffer algorithm with mesh triangles representation in barycentric coordinates is employed. The proposed recognition method is tested and experimentally validated in several real world case studies.
\end{abstract}

Index Terms-3D vision, Industrial automation systems, Intelligent manufacturing systems, Manufacturing automation, Object recognition, Object segmentation, Reverse engineering

\section{INTRODUCTION}

$\mathrm{L}$ ed by extensive development of 3D data acquisition $\Lambda_{\text {systems, the field of application of reverse engineering }}$ (RE) techniques has significantly expanded over the recent years. The speed, accuracy and mobility of the state of the art scanning devices [1], [2], especially optical ones, gave a new momentum for implementation of RE methods in manufacturing process control [3] and inspection [4]. In intelligent manufacturing systems, which should be able to

Manuscript received Jun 23, 2014; accepted for publication December 19 , 2014.

Copyright (C) 2009 IEEE. Personal use of this material is permitted. However, permission to use this material for any other purposes must be obtained from the IEEE by sending a request to pubs-permissions@ieee.org

This work was supported in part by Serbian Ministry of Education, Science and Technological Development, research grants TR35007, TR35020, TR35022. The authors express gratitude to CAD Professional Systems doo, Belgrade, and Topomatika Zagreb for scanning service.

Z. Jakovljevic and R. Puzovic are with University of Belgrade, Faculty of Mechanical Engineering, Department for Production Engineering, Kraljice Marije 16, 11000 Belgrade, Serbia (e-mail: zjakovljevic@mas.bg.ac.rs; rpuzovic@mas.bg.ac.rs).

M. Pajic is with the Department of Electrical and Computer Engineering, Duke University, Durham, NC 27708 USA (email: miroslav.pajic@duke.edu). adapt to changing environments full of uncertainties, 3D scanning devices provide indispensable feedback information that can be used for control of tasks carried out by robots, handling equipment, assembly systems, etc.

These industrial applications have imposed new requirements to 3D digital acquisition systems, such as large measuring volume along with satisfactory precision, high speed, real-time data processing, reliability in real-world industrial environments, and all of this at affordable prices. Whereas hardware in modern devices provides high speed and resolution, the bottleneck for their use in real-time is data processing [5]. The raw 3D data registration, integration, and meshing have been in the focus of many research studies, resulting in generation and implementation of a number of very successful techniques for solving these problems [5], [6]. Nevertheless, point cloud segmentation, simplification and surface fitting still remain the most critical elements of these systems and an open area for research (this has been thoroughly addressed in the related work section).

The generation of a dimensionally correct polygonal mesh [7] from a point cloud is a standard feature of CAD (Computer Aided Design) systems. However, the resulting 3D models, although aesthetically sound, are not composed of high level geometric primitives (e.g., planes, natural quadrics) present in the initial object model, and thus do not show the original designer's intent. The reconstruction of high-level parametric part geometry from a point cloud is typically carried out manually through annotation of points that belong to certain parametric surfaces [8]. This task is very tedious, time consuming, and error prone.

On the other hand, automatic point cloud segmentation and surface fitting as well as the reconstruction of object's primary geometry are the main requirements for implementation of 3D scanning devices in automated manufacturing of scanned objects and in real-time control of manufacturing processes in industrial conditions. Typical example is recognition of planes in a scene during motion planning in mobile robotics, which is a very important issue within reconfigurable manufacturing systems [9]. Another example is detection of the seam pose in robotized welding tasks [3], [10], in unstructured or semistructured environments. Furthermore, automatic workpiece alignment in machining systems as well as teleoperation using augmented reality [11] would extensively benefit from the use of 3D scanning devices and recognition of the primary geometry.

Segmentation and fitting of planes in point cloud has attracted more research efforts than other types of primary 
geometry. Besides the simplicity of the planar surface, there are other mainly application driven reasons for this. For example, online navigation of mobile robots [12]-[14] in unstructured environment using sensory information from triangulation laser scanners [15] or structured light systems [1], [16] requires recognition of planar surfaces (e.g., walls, machines and other objects in robot's surroundings) from point cloud [17], [18]. Another application domain for plane recognition is automatic architectural reconstruction [19]-[21].

In this paper, we propose a fast one-pass algorithm for extraction/segmentation of planar regions from a point cloud that is based on Discrete Wavelet Transform (DWT). Note that due to its convenient properties wavelet transform has been extensively employed for 2D image analysis, showing excellent results in e.g., image compression [22], [23], denoising [24], texture analysis [25]. However, the use of DWT in 3D point cloud processing is relatively new and thus far very limited. Some recent papers reported the utilization of wavelet transform in analysis of 3D geometric texture [26], scattered point cloud structuring [27] and compression [28], as well as in 3D point clouds integration [29]. Nevertheless, to the best of our knowledge, except in our research, wavelet transform has not been previously employed in recognition of planar segments in a point cloud.

In this paper, we have opted to employ DWT in order to exploit the orthogonality of Daubechies wavelets to polynomials for segmentation of planar regions from point cloud (we will elaborate on this in Section 4). Advantageous properties of DWT, i.e., the combination of its high computational speed and insensitivity of certain wavelets to linear functions, provide a solid basis for creation of fast plane segmentation algorithm. We show that after efficient segmentation of planar regions, their parameters can be easily estimated using different fitting procedures (e.g., least squares regression). The advantages of our algorithm over existing methods for planar segments recognition from a point cloud (these methods will be addressed in the next section) are its simplicity, speed (planes are recognized in a single pass), the fact that the method is autonomous (i.e., there is no need for user intervention), and the precision of estimated plane parameters is data driven. We illustrate these advantages and real-world applicability of the proposed procedure on three case studies. We effectively show that the proposed algorithm outperforms the existing algorithms for planar segment detection in the execution time. First case study is a frequently used benchmark test piece; second considers a test piece with known geometry, whereas in the third case study we recognize the planar segments in a test piece with unknown and extremely complex geometry from the plane recognition point of view.

The rest of the paper is organized as follows. Related work is discussed in Section 2. Section 3 presents relevant theoretical background, while the proposed method for recognition of planar regions in a point cloud is presented in Section 4. In Section 5, we apply the proposed procedure to three practical case studies. Finally, Section 6 presents concluding remarks and avenues for future work.

\section{RELATED WORK}

Currently, there are three main approaches for recognition of planar features in a point cloud, based on 3D Hough transform, RANSAC (Random Sample Consensus) method, and region growing.

3D Hough transform [17] is an extension of 2D Hough transform [30] that maps each plane from $\mathrm{R}^{3}$ space into a single voxel in the discrete Hough space whose coordinates are plane parameters $(\theta, \varphi, r)$. Using this procedure, every point $\mathbf{P}(\mathrm{x}, \mathrm{y}, \mathrm{z})$ from the original $\mathrm{R}^{3}$ space is mapped into a large number of voxels in Hough space $(\theta, \varphi, r)-$ a voxel for each plane that passes through the $\mathbf{P}(\mathrm{x}, \mathrm{y}, \mathrm{z})$. The planes in a point cloud are recognized using a voting procedure. The voxel that contains the highest number of votes (points mapped from the point cloud) represents the plane in $\mathrm{R}^{3}$ space. If there is more than one plane in $\mathrm{R}^{3}$ space there will be more voxels with a high number of votes - one voxel for each plane. On the other hand, if there are no planes in $\mathrm{R}^{3}$ space, the points will be scattered all over the space. The threshold for detection of planes has to be fine-tuned to detect small planar segments and yet not to be prone to detection of nonexistent planes. Besides, there is a tradeoff between the velocity of execution and the accuracy of plane parameters that can be tuned by Hough space quantization. To precisely determine plane parameters, the space discretization steps should be small leading to higher computational costs.

RANSAC [31] randomly selects a minimal set of points from a point cloud and calculates free parameters of the model (i.e., geometric primitives). In the case of a plane, it picks 3 points and fits the candidate plane through them. When parameters of the primitive candidate are estimated, the algorithm detects the points from the cloud that belong to this primitive and the procedure is repeated for a predefined number of times. The geometric primitive that contains the highest number of points is considered to be the best fit for given data. The original RANSAC method assumes that only one model can be fit through a point cloud, e.g., one plane. If there is more than one geometric primitive in the cloud, the points that belong to the first detected primitive are removed from the cloud, and the algorithm is recursively applied until all the points are exhausted. In this case, the computational cost of the algorithm can be very high due to the randomness of points sampling. To deal with this issue, in [32] the authors propose localized sampling strategy that considers the distance between the points. Nevertheless, the proposed strategy is based on computation of a surface normal vector for each point and requires a number of parameters that have to be finetuned for fast performance and precise results.

There are a number of region growing methods that start from a few points from the cloud and construct regions by expanding the seed according to parameters of geometric primitives. Different clustering methods have been proposed, including k-means clustering [33], agglomerative hierarchical clustering of the mesh triangles [34], [35] or points from the cloud [36], partition based on expectation maximization [37], multidimensional particle swarm optimization [38], detection 
of peaks in spherical coordinates [39], and estimation of subwindow plane parameters [40]. These methods usually utilize the best fit of geometric primitives as a metric, assuming that the possible primitives are known a priori. Selection of the initial seed is highly critical for the performance of region growing methods, and it is usually performed manually.

To address these limitations, we present a new algorithm for recognition (segmentation and fitting) of planar regions from a point cloud. The proposed algorithm segments all planes visible from a selected viewpoint in one pass. The accuracy of estimated plane parameters using our method is data driven. Furthermore, the method is not based on region growing, it is autonomous, and does not require interventions from the user.

\section{OVERVIEW OF DISCRETE WAVELET TRANSFORM}

The method for recognition of planar regions that we propose in this paper employs Discrete Wavelet Transform (DWT). Before presenting the proposed procedure, in this section, we briefly introduce the background of DWT.

Wavelet transform represents a one-dimensional signal $f(t)$ as a linear superposition of atomic functions called wavelets. The wavelets are obtained by translation and dilatation of a single non-periodic function - the mother wavelet. In discrete wavelet transform, mother wavelet is translated and dilated with discrete steps. DWT of signal $f(t)$, denoted by $T_{m, n}$, can be represented by the following equation [41]:

$$
T_{m, n}=\int f(t) a_{0}^{-m / 2} \overline{\psi\left(a_{0}^{-m / 2} t-n b_{0}\right)} d t
$$

Here, $\psi(t)$ is the mother wavelet, $a_{0}{ }^{m}$ is dilatation, and $b_{0}$ translation step, while overline denotes complex conjugate operation. The presented discretization is non-uniform in timefrequency space, and it provides a higher time resolution for low and higher frequency resolution for high frequencies. To make DWT unique and inverse DWT feasible, the wavelets used in DWT must form an orthonormal basis [41].

Multiresolution analysis (MRA), originating from image processing, has had a significant impact on DWT. Besides subband filtering scheme [42], which is a fast real-time applicable hierarchical algorithm for execution of direct and inverse DWT, MRA provides efficient methods to generate orthonormal wavelet bases. The basic MRA algorithm for DWT can be briefly stated as follows ${ }^{1}$. It starts from a sequence of resolutions $2^{-j}, j \in(0,-\infty)$. Approximation of signal $f$ at the resolution $j=1$ is denoted by $A_{l}$. This approximation is obtained at the first level of transform by subtracting details $D_{l}$ from the signal $f$. Similarly, approximation $A_{2}$ is obtained by subtracting details $D_{2}$ from approximation $A_{l}$. This process is repeated until the $J^{\text {th }}$ level of transform. Following this procedure, the signal $f$ can be represented as a sum of its approximation at resolution $J$ (i.e., $\left.A_{J}\right)$ and details $D_{j}, j \in[1, J]$ taken from it during passing from

\footnotetext{
${ }^{1}$ Due to lack of space, in this section we provide a high-level description of the MRA and subband filtering. Detailed description of MRA and subband filtering scheme used to compute details and approximations $D_{j}$ and $A_{j}$, can be found in [41], [42].
}

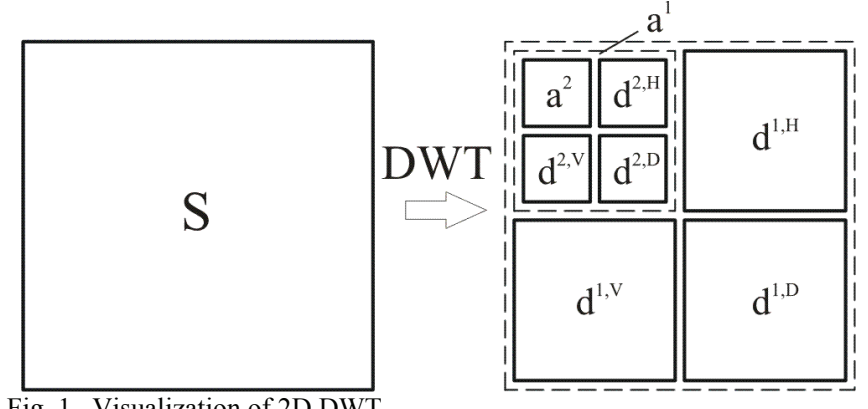

Fig. 1. Visualization of 2D DWT.

higher level of approximation (resolution) to the lower one

$f=A_{J}+\sum_{j=1}^{J} D_{j}=\underbrace{\sum_{n} a_{n}^{J} \phi_{J, n}}_{A_{J}}+\sum_{j=1}^{\sum_{j} \underbrace{\sum_{n} d_{n}^{j} \psi_{j, n}}_{D_{j}}}$

In the above equation, orthonormal bases $\left\{\psi_{j, n}, n \in Z\right\}$ and $\left\{\phi_{J, n}, \quad n \in Z\right\} \quad$ represent the family of wavelets and corresponding scaling functions, $a_{n}^{J}$ are the approximation and $d_{n}^{j}$ the detail coefficients, computed by the aforementioned subband filtering scheme [42]. Finally, $J$ denotes the level of transform.

There are a number of families of wavelets (Daubechies wavelets, coiflets, symmlets, biorthogonal wavelets [41]) that form orthonormal bases. All of them are compactly supported, thus providing excellent time/spatial localization properties to the transform. In addition, some of them have an asymmetric shape and can be employed for detection of abrupt changes in signal such as edges.

Besides application in time series analysis, DWT can be performed on two dimensional signal represented by matrix $\mathrm{S}$ with dimensions $m \times n$, using the following procedure:

Step 1: One-dimensional DWT of each row of matrix $S$

Step 2: One-dimensional DWT of columns computed in step 1.

Two-level DWT of two-dimensional signal $\mathrm{S}$ can be visualized [41] as shown in Fig. 1. Here, $a^{i}$ represent approximation and $d^{i, H}, d^{i, V}$ and $d^{i, D}$ detail coefficients at $i=1$, 2 levels of transform. These matrices have $2^{n}$ (where $n$ is the level of transform) smaller dimensions then the original signal matrix. Two dimensional DWT inherits all the properties of one dimensional DWT including real time applicability and sensitivity to abrupt changes.

\section{Method for Recognition of Planar Regions Based ON DWT}

To detect planar regions in a point cloud, we exploit the orthogonality of certain wavelets to polynomial functions, in particular to constant and linear functions. After effective detection, we estimate plane parameters by using standard fitting procedures. In this section, before presenting the method itself, we introduce some theoretical background and present the idea on a synthesized surface.

\section{A. Vanishing Moments of Wavelets}

Besides suitability for detection of abrupt changes (e.g., edges) in a signal, there exists another property of certain 
a)

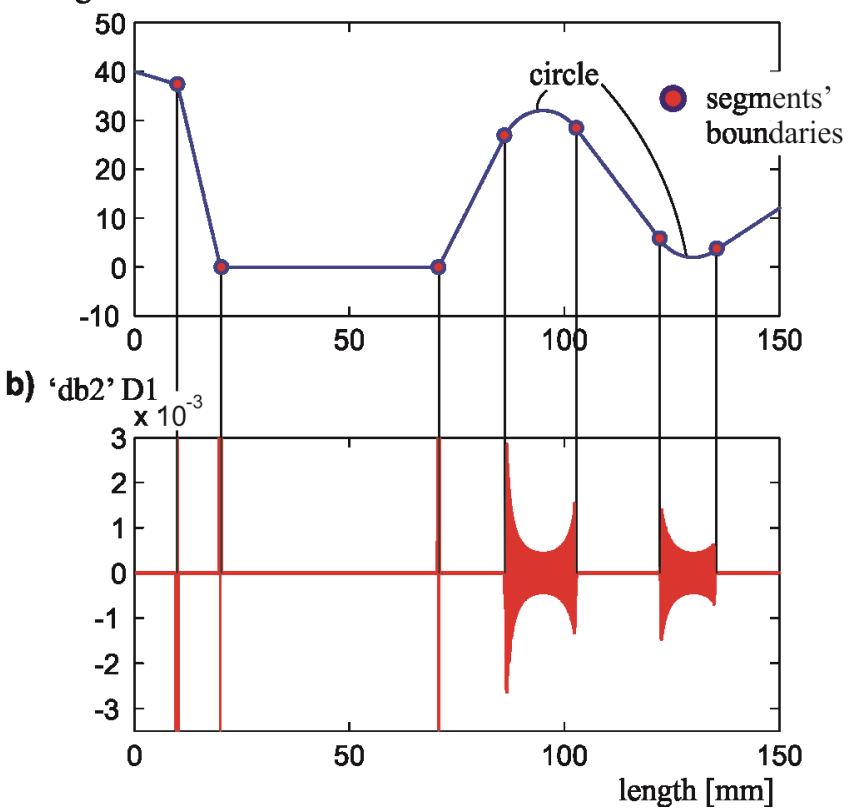

Fig. 2. Synthesized 2D signal: a) Original signal, b) The details of the first level DWT using 'db2'.

wavelet families that is crucial for the detection of planar regions in point cloud. Namely, the wavelets from Daubechies family ( $\mathrm{db}$ wavelets) have $\mathrm{N}$ vanishing moments [41], meaning that:

$\int x^{n} \psi(x) d x=0, \quad n=0,1, \ldots N-1$

where $\mathrm{N}$ denotes the order of wavelet $\psi$. Thus, the $\mathrm{db}$ wavelets of order $\mathrm{N}$ are orthogonal to the polynomials $1, x, x^{2}, \ldots x^{\mathrm{N}-1}$ and the signal, after DWT is applied, will be approximated by polynomials of order N-1. Consequently, the detail coefficients in the areas of the signal that are well approximated by a polynomial of order $\mathrm{N}-1$ will be close to zero. In the case of Daubechies wavelet of order 2 (i.e., db2 wavelet), the detail coefficients and details will be close to zero in all areas in which a signal is well approximated by a linear function or a constant. On the other hand, in all nonlinear areas, the detail coefficients will have large values. It is very important for the application at hand that the detail coefficients will extract high frequency noise from real world signals [43] and that the presented approach for detection of linear segments is not sensitive to this kind of noise.

Furthermore, on a transition between two $C^{0}$ continuous $^{2}$ segments (e.g., linear segments with different inclination), there will be an abrupt change in signal. Since db2 is a highly asymmetric wavelet, it will be sensitive to this change and the level of detail coefficients at the connection of two $C^{0}$ continuous segments will be high. To illustrate this, Fig. 2 presents the first level details obtained by decomposition of a synthesized signal using db2 wavelet. The signal (Fig. 2a) consists of six linear and two circular segments. The details of the signal at the first level of DWT using $\mathrm{db} 2$ wavelet are shown in Fig. 2b. It can be seen that details are equal to zero

\footnotetext{
${ }^{2}$ Values of functions representing segments are equal in their intersection point, but the values of higher derivatives of functions are not equal
}

in all areas in which the signal is constant or linear, while details differ from zero in areas corresponding to circular segments. The boundaries between segments represent abrupt changes in the signal and DWT detects them - i.e., details differ from zero. This information is very useful for segmentation of adjacent linear segments. Although the transition between linear and circular segments is $G^{1}$ continuous $^{3}$, and there is no abrupt change, the increase of detail coefficients at segments boundaries is present due to the different polynomial representation of adjacent segments.

\section{B. Planar Segments Detection using DWT}

Two-dimensional DWT inherits all properties of the onedimensional transform including sensitivity to abrupt changes (edges) and orthonormality to polynomial surfaces. These are the properties of $\mathrm{db}$ wavelets that we exploit for generation of
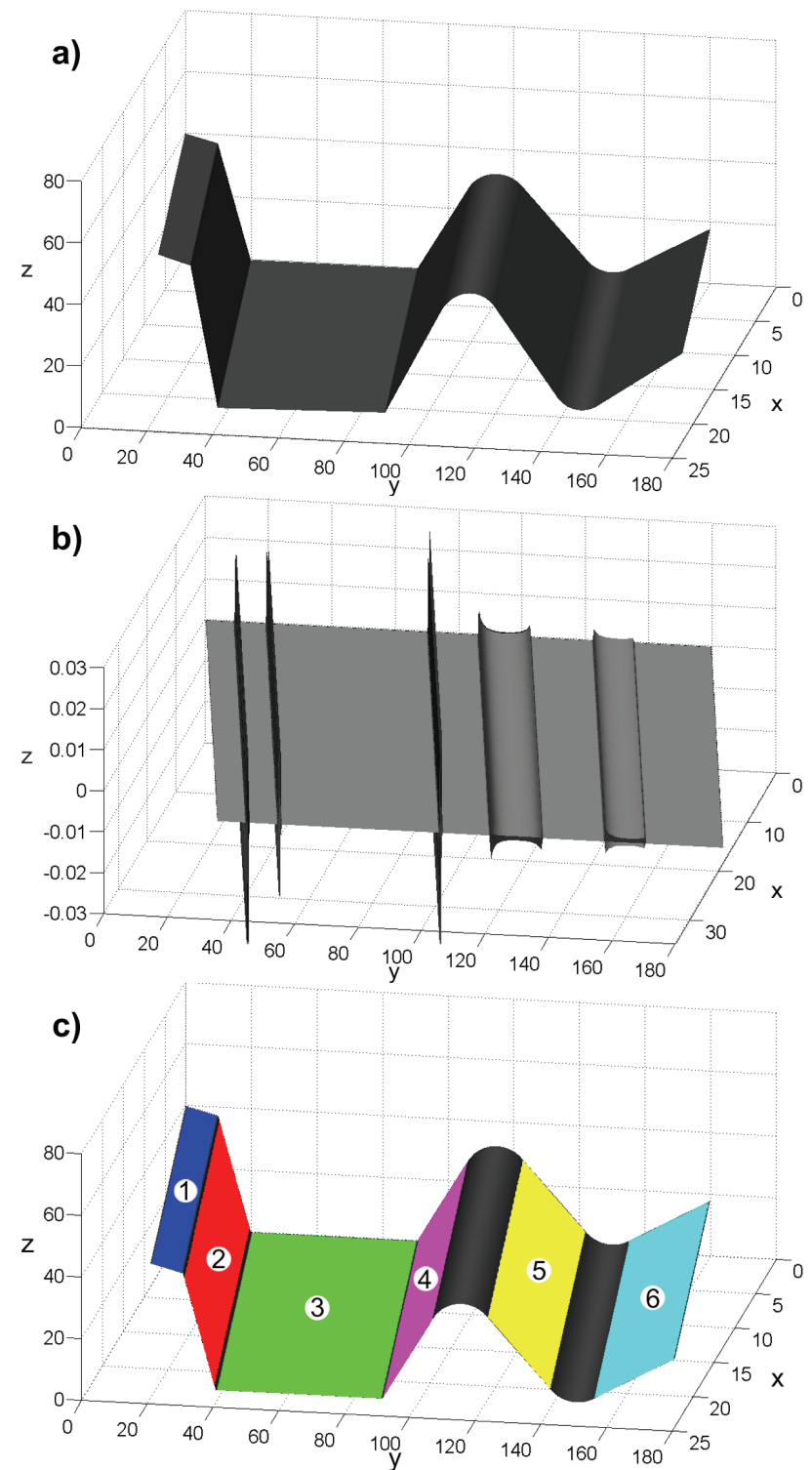

Fig. 3. Synthesized 3D signal (point cloud): a) Original signal, b) The details of the first level 2D DWT obtained using ' $\mathrm{db} 2$ ', c) The fitted planes obtained using the proposed procedure.

\footnotetext{
${ }^{3}$ Tangent vectors of functions have the same direction in intersection point
} 
a)

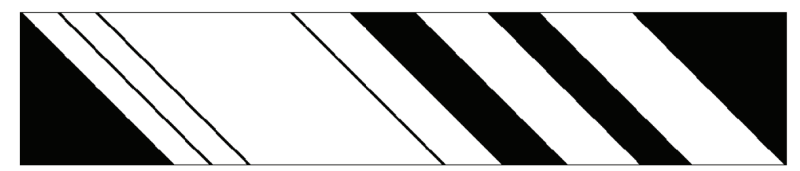

b)

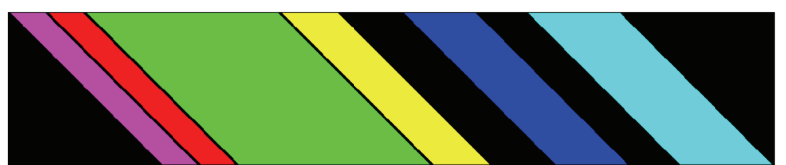

Fig. 4. a) The binary 2D matrix after thresholding of the signal presented in Fig. 3b, b) Recognized connected regions in binary image.

TABLE I

Normal Vectors and Points on Detected Planar SegMents OBTAINED USING THE PROPOSED DWT-BASED METHOD FROM THE SYNTHESIZED SIGNAL SHOWN IN FIG. 3A

\begin{tabular}{lll}
\hline \hline $\begin{array}{l}\text { Planar } \\
\text { segm. }\end{array}$ & \multicolumn{1}{c}{ Normal vector } & \multicolumn{1}{c}{ Point on the plane } \\
\hline 1 & {$[0.7746,-0.1637,-0.6109]$} & {$[-31.5259,149.1820,39.9732]$} \\
2 & {$[0.7746,-0.6109,-0.1637]$} & {$[-15.6946,19.9000,74.2678]$} \\
3 & {$[0.7071,0.0000,-0.7071]$} & {$[0.0000,89.2234,0.0000]$} \\
4 & {$[-0.3437,0.8133,-0.4695]$} & {$[-167.1627,70.6513-122.3716]$} \\
5 & {$[0.8157,-0.4384,-0.3774]$} & {$[-68.2649,127.0357,147.5578]$} \\
6 & {$[0.3437,0.4695,-0.8133]$} & {$[176.3748,129.1153,-74.5448]$} \\
\hline \hline
\end{tabular}

a fast one-pass algorithm for segmentation of planar regions. Namely, the details of 2D DWT using db2 wavelet will be close to zero in all areas that represent planar surfaces, and we utilize this fact to segment planar regions from a point cloud.

To illustrate the segmentation of planar regions using 2D db2 DWT, we synthesized a surface that consists of six planar and two cylindrical segments (Fig. 3a). This surface is generated by extrusion of the profile presented in Fig. 2 along the vector $[1,1,1]$. Fig. $3 \mathrm{~b}$ presents the details of $2 \mathrm{D} \mathrm{db} 2$ DWT at the first level of transform of the selected surface. As expected, the details are equal to zero in points that belong to planar segments. The only exceptions are the points at the surface edges. These high level details will be exploited for the segmentation of adjacent planar segments. Simple thresholding of the details (Fig. 3b), when observed from positive $\mathrm{z}$-axis, leads to generation of a binary $2 \mathrm{D}$ matrix (binary image) that contains one connected region for each planar segment in the processed surface (Fig. 4a).

Using the standard procedure [44] for detection of connected components in 2D binary image (Fig. $4 \mathrm{~b}$ ), and then returning into the $3 \mathrm{D}$ space, the planar segments are recognized and segmented. After the segmentation, since all data that belong to one segment are sampled from a single plane, we can apply standard procedures (e.g., least squares regression) for identification of plane parameters. The detected planar segments are shown in Fig. $3 \mathrm{c}$, and the estimated plane parameters in Table I. Estimated plane parameters are exactly the same as the synthesized surfaces' parameters, since the considered signal is without noise. For example, the unit vector of the cross product between the direction vector of profile line of segment 3 ([ $\left.\left[\begin{array}{lll}0 & 1 & 0\end{array}\right]\right)$ and the extrusion vector $\left(\left[\begin{array}{lll}1 & 1 & 1\end{array}\right]\right)$ is $[0.7071,0.0000,-0.7071]-$ exactly the value detected by the proposed method (Table I). Note that the angles between synthesized profile lines of segments 1-6 and y-axis are $-15^{\circ},-75^{\circ}, 0^{\circ}, 60^{\circ},-49.2741^{\circ}$, and $30^{\circ}$, respectively. The performances of the procedure on real world signals will be addressed in the next section.

\section{Algorithm for Detection of Planar Segments}

As a result of the previous observations, we propose a method for segmentation of planar segments that can be summarized by Algorithm 1. After the application of the given procedure all the points belonging to planes will be clustered.

The presented algorithm computes one level 2D DWT of the signal using $\mathrm{db} 2$ wavelet. The subband filtering scheme used to implement this transform consists of a convolution of the signal by a filter with 4 samples, and downsampling of the signal by 2 for each row and column of the starting 2D matrix (to obtain detail coefficients $d^{1, H}, d^{1, V}$ and $d^{I, D}$ ). In addition, details $D_{l}$ are computed using inverse DWT on detail coefficients (approximation coefficients $a^{i}$ are equal to zero). The inverse DWT requires signal upsampling by 2 and convolution by a filter with 4 samples for each row and column. Consequently, the presented method is computationally inexpensive. In addition, all planar regions in the signal are detected in a single pass, which is a significant advantage of this method to the RANSAC algorithm (since RANSAC is iterative when more than one plane is present).

As shown in Table I, the accuracy of estimated plane parameters is data driven. For a signal without noise, the method is able to estimate the exact value of parameters. On the other hand, the estimate of plane parameters using 3D Hough transform would depend on the discretization of Hough space.

Algorithm 1 Recognition of planar regions from point cloud

Step 1: 2D DWT of the scanned surface using $\mathrm{db} 2$ and generation of $D_{l}$ details

Step 2: Thresholding of $D_{l}$ details with the predefined threshold

Step 3: Generation of binary image

Step 4: Segmentation of $\mathrm{k}$ connected regions in the binary image

for $i=1: k$

Step 5: Selection of points from point cloud whose projection on xy plane is in the corresponding $2 \mathrm{D}$ region

Step 6: Fitting the plane through points and merging fitted planes with identical parameters

Step 7: From the point cloud, select all the points whose projection is with predefined tolerance within the corresponding 2D region and that belong to the fitted plane within predefined interval (tolerance); denote selected points as points on the plane $\mathrm{i}$

end

It is worth noting that the proposed method does not require user intervention during the plane recognition process. The only parameter that has to be set before the recognition process starts is the signal details threshold. The appropriate value of the threshold depends on the roughness and waviness 
of scanned surface and it should be specified for the application at hand. Note that the surface finish of scanned objects is usually uniform for certain classes of engineering tasks (e.g., for sheet metal, castings, and machined parts). Thus, when a threshold is adequately set once, there will be no need for its frequent change. In the case studies presented in the next section, we have estimated the threshold by taking a convenient sample of a planar region. We performed one level 2D DWT on this sample, and estimated the threshold as a maximum of absolute values of details $D_{l}$. This value can be observed as a roughness and waviness of selected planar region. As part of our future efforts we plan to investigate robustness of the proposed threshold assignment procedure to extremely rough surfaces.

The presented method detects planar surfaces whose normals' inclination to positive $\mathrm{z}$-axis is in the $\left(-90^{\circ}, 90^{\circ}\right)$ range. This is due to the selection of $z$-axis positive half as the primary direction and xy-plane as the basis for 2D DWT application. For detection of the surfaces with different inclination angles, the procedure should be repeated by taking $-\mathrm{z}, \pm \mathrm{y}$ and $\pm \mathrm{x}$ directions as primary and corresponding planes as the bases for 2D DWT application. If some regions are visible from two or three directions, the parameters of fitted planes from different directions will be identical (if there is no noise in the signal) and this information can be used for merging the same regions. Alternatively, the points belonging to a single plane can be excluded from the point cloud before applying the procedure in other directions.

It should be also emphasized that the presented procedure requires a structured point cloud at input. Essentially, the most of the available scanning devices inherently have the structured point cloud as the low level output and scan the surface only in the positive $\mathrm{z}$ direction [2]. However, due to subsequent processing such as multiple view integration, a number of 3D scanners provide an unstructured point cloud as the final output. In such cases, a procedure for point cloud structuring should be conducted prior to the use of the presented method for segmentation of planar regions. Alternatively, low-level output can be used in control tasks.

\section{REAL WORLD APPLICATIONS}

We have applied the presented method for recognition of planar segments in a point cloud to three real world industrial case-studies. The first example is a benchmark used in various works on reverse engineering [45]-[48]. The second case study focuses on a part bounded with planar surfaces, while the third example deals with an insert of injection molding die.

All the parts were scanned using the ATOS Compact Scan 3D scanning device by GOM GmbH [49]. The scanning device provides an unstructured point cloud in STL format with a very high resolution. The parts were scanned in multiple directions. To obtain a structured scan of the surface in the positive $\mathrm{z}$ direction, we have employed a procedure based on the $\mathrm{z}$ buffer algorithm [50] and representation of triangles in barycentric coordinates.
To illustrate the performance of the proposed method we have compared its computational cost, results, and parameter initialization with standard 3D Hough transform using algorithm from [17] and RANSAC using code provided in [51]. All results presented in this section have been obtained when the algorithms have been tested on a system with 1.7 $\mathrm{GHz}$ Intel Core i7 processor (with 2 cores) and $8 \mathrm{~GB}$ of 1600 $\mathrm{MHz}$ DDR3 memory. Finally, we discuss the obtained results at the end of the section.

\section{A. Case Study 1 - Planar Surfaces Parallel to Coordinate Axes}

In this case study we analyze the benchmark part from Fig. 5 , which was made on a 3-axis machining center and has very poor surface finish. After scanning, point cloud contained 792,522 points, which gave a very high resolution on a part with $26.5 \times 50 \times 90 \mathrm{~mm}$ dimensions. Before applying the proposed segmentation procedure we have re-sampled the point cloud and extracted the surface visible from the positive $\mathrm{z}$ axis. After transforming the extracted surface with one level 2D db2 DWT and thresholding the details, the proposed method generated the binary image presented in Fig. 6a. Fig. $6 \mathrm{~b}$ shows the recognized connected regions in the binary image. Following the algorithm presented in Section 4 along $+z$ axis, we have obtained 3 planar segments, whose plane parameters are given in Table II (segments 1-3). Fig. 7a presents mesh triangles on the points from the cloud that were recognized as points belonging to the fitted planes.

The procedure was repeated for the surfaces visible from the $\pm \mathrm{x}$ and $\pm \mathrm{y}$ directions, and the recognized planar regions are presented in Fig. 7b. Fig. 7 illustrates that the proposed procedure efficiently segmented planar segments, regardless the very inconvenient surface texture. In this case study all planar surfaces on the test piece were parallel to the coordinate axes, which is in accordance with parameters of recognized planes from Table II. Namely, all the normal vectors presented in this table are parallel to coordinate axes as expected. The error is less than $0.15 \%$.

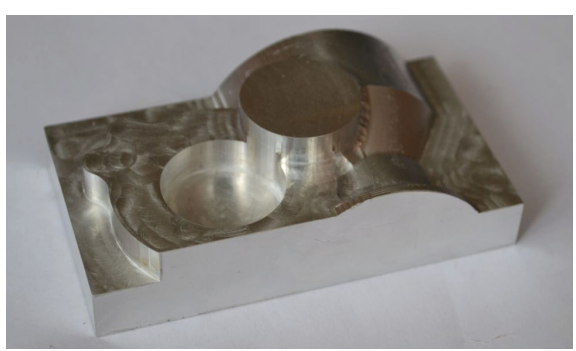

Fig. 5. The test piece used in case study $1-$ machined part with planar surfaces parallel to coordinate axes.
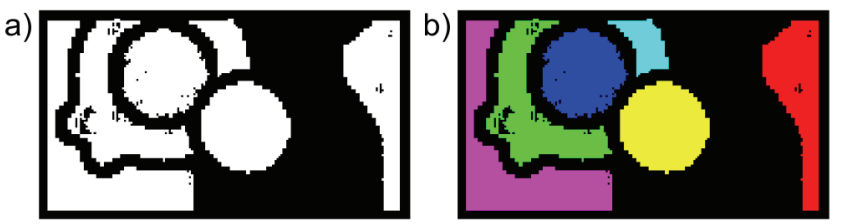

Fig. 6. a) Binary image after details thresholding; b) Detected connected regions in binary image. 
a)

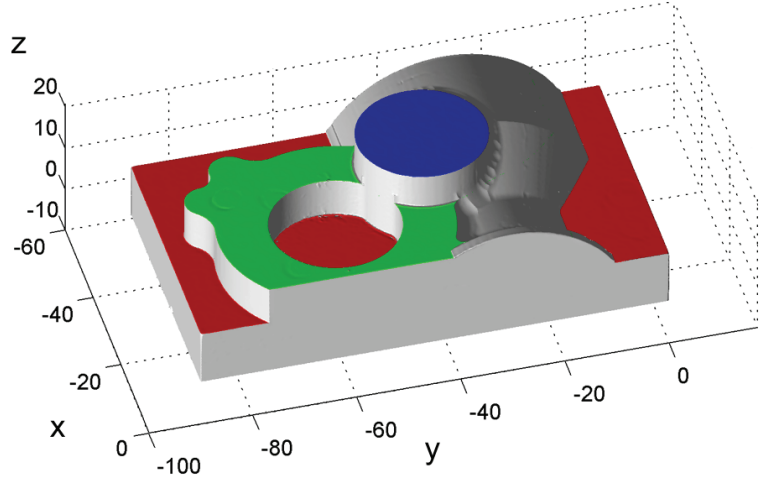

b)

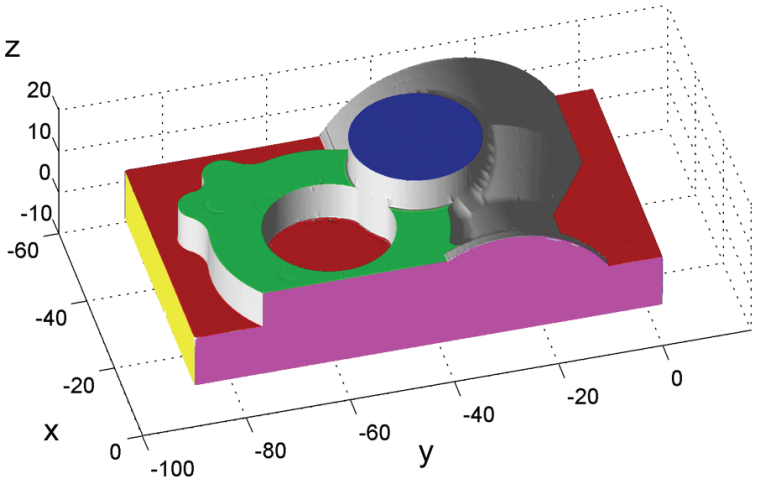

Fig. 7. a) Detected planar regions visible from positive $z$ axis; b) Detected planar regions on the scanned part.

TABLE II

Normal Vectors and Points on Detected Planar Segments OBTAINED BY ALGORITHM 1 In CASE STUdY 1

\begin{tabular}{llll}
\hline \hline \multirow{2}{*}{$\begin{array}{c}\text { Planar } \\
\text { segm. }\end{array}$} & \multicolumn{2}{c}{ Normal vector } & \multirow{2}{*}{$\begin{array}{c}\text { Point on the } \\
\text { plane }\end{array}$} \\
\cline { 2 - 3 } & Cartesian coordinates & Spherical coord. & \multicolumn{1}{c}{} \\
\hline 1 & {$[-0.0006,-0.0002,1.0000]$} & {$\left[1,-161.57^{\circ}, 0.00^{\circ}\right]$} & {$[0,0,11.48]$} \\
2 & {$[-0.0004,-0.0001,1.0000]$} & {$\left[1,-165.96^{\circ}, 0.00^{\circ}\right]$} & {$[0,0,19.49]$} \\
3 & {$[-0.0011,-0.0001,1.0000]$} & {$\left[1,-174.81^{\circ}, 0.00^{\circ}\right]$} & {$[0,0,26.52]$} \\
4 & {$[-0.0002,1.0000,0.0006]$} & {$\left[1,90.01^{\circ}, 89.97^{\circ}\right]$} & {$[0,0.00,0]$} \\
5 & {$[0.0000,-1.0000,-0.0004]$} & {$\left[1,-90.00^{\circ}, 90.02^{\circ}\right]$} & {$[0,-90.04,0]$} \\
6 & {$[1.0000,-0.0000,0.0000]$} & {$\left[1,-0.00^{\circ}, 90.00^{\circ}\right]$} & {$[0.00,0,0]$} \\
7 & {$[-1.0000,0.0001,-0.0001]$} & {$\left[1,179.99^{\circ}, 90.01^{\circ}\right]$} & {$[-50.02,0,0]$} \\
\hline \hline
\end{tabular}

TABLE III

RESULTS OF COMPARISON OF ALTERNATIVE ALGORITHMS IN CASE STUDY 1

\begin{tabular}{|c|c|c|c|c|}
\hline Algorithm & $\begin{array}{c}\mathrm{CPU} \\
\text { time [s] }\end{array}$ & Parameter & $\begin{array}{l}\text { Value of } \\
\text { parameter }\end{array}$ & $\begin{array}{c}\text { Detected plane } \\
{[\mathrm{r}, \theta, \varphi]^{\mathrm{a}}}\end{array}$ \\
\hline DWT based & 0.45 & Detail thresho & 0.002 & {$\left[26.52,-174.81^{\circ}, 0.00^{\circ}\right]$} \\
\hline 3D Hough & 118.15 & $\begin{array}{l}\text { Res. }(\theta, \varphi, r) \text { : } \\
\text { Threshold: } \\
\text { Range for } r \\
\text { No. of planes }\end{array}$ & $\begin{array}{l}5^{\circ}, 5^{\circ}, 0.5 \mathrm{~mm} \\
0.2 \mathrm{~mm} \\
0 \div 27 \mathrm{~mm} \\
3\end{array}$ & {$\left[26.5,-180^{\circ}, 0^{\circ}\right]$} \\
\hline RANSAC & 2.71 & $\begin{array}{l}\text { Threshold } \\
\text { No. of planes }\end{array}$ & $\begin{array}{l}0.2 \mathrm{~mm} \\
3\end{array}$ & {$\left[26.52,-177.95^{\circ}, 0.07^{\circ}\right]$} \\
\hline
\end{tabular}

${ }^{a}$ An example of detected plane

For the surface visible from positive z-axis besides our method, we have employed 3D Hough transform and RANSAC algorithm for detection of planar regions. The computational cost and a sample of recognized plane along with algorithms' parameters are presented in Table III.

\section{B. Case Study 2 - Planar Surfaces with Arbitrary Orientation}

In the previous case study, all planar surfaces were mutually perpendicular or parallel. The scanning procedure was performed in a way that all planar regions were perpendicular to one of the coordinate axes. In this case study, we have used a test piece bounded by planes with different orientations. The scanned part is presented in Fig. 8. The surface finish of the part is very poor, even worse than in the previous case study.

The part was scanned and the obtained point cloud was very dense with 1,059,619 points on the part with dimensions ca. $48.30 \times 70 \times 100 \mathrm{~mm}$. After carrying out the complete procedure for surfaces visible from the $+\mathrm{z}, \pm \mathrm{x}$ and $\pm \mathrm{y}$ directions, we have recognized the boundary planes with parameters presented in Table IV. The plane parameters are in accordance with the part geometry since the part was designed in such a way that the angles between base planar surface and inclined planar surfaces were $20^{\circ}, 45^{\circ}$ and $60^{\circ}$. The error between designed and detected plane orientations is less then $1 \%$. Note that the

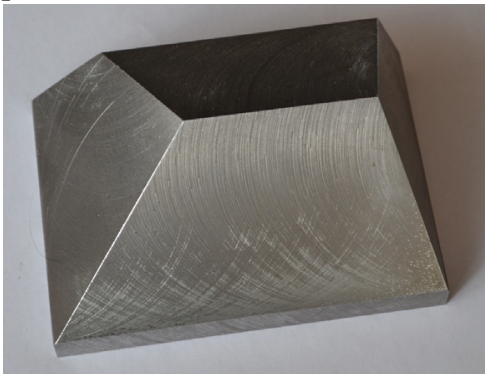

Fig. 8. The test piece used in case study 2 - machined part with planar surfaces with arbitrary orientation.

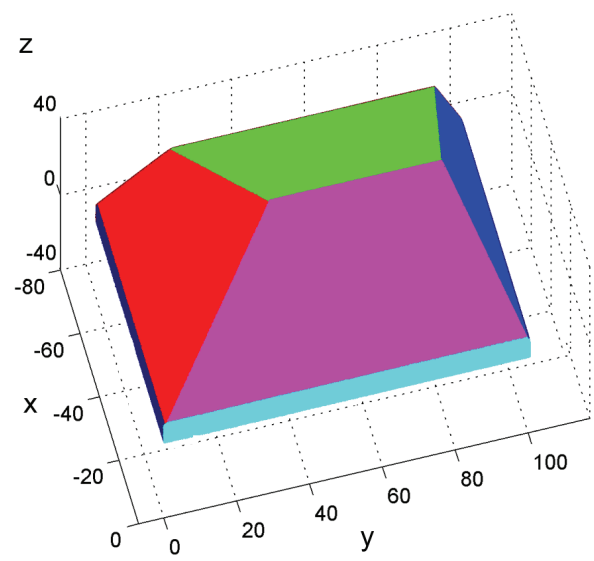

Fig. 9. Detected planar regions in the test piece with planar segments with arbitrary orientations.

TABLE IV

Normal Vectors and Points on Detected Planar SEgments OBTAINED BY ALGORITHM 1 IN CASE STUDY 2

\begin{tabular}{cccc}
\hline \hline \multirow{2}{*}{$\begin{array}{c}\text { Planar } \\
\text { segm. }\end{array}$} & \multicolumn{2}{c}{ Normal vector } & \multirow{2}{*}{$\begin{array}{c}\text { Point on the } \\
\text { plane }\end{array}$} \\
\cline { 2 - 4 } & Cartesian coordinates & Spherical coord. & \multicolumn{1}{c}{ S } \\
\hline 1 & {$[-0.0023,-0.7062,0.7080]$} & {$\left[1,-90.19^{\circ}, 44.93^{\circ}\right]$} & {$[-40,50,58.68]$} \\
3 & {$[-0.4969,0.0001,0.8678]$} & {$\left[1,179.99^{\circ}, 29.80^{\circ}\right]$} & {$[-40,50,46.66]$} \\
4 & {$[0.0011,0.9408,0.3389]$} & {$\left[1,89.93^{\circ}, 70.19^{\circ}\right]$} & {$[-40,50,148.99]$} \\
5 & {$[0.7104,-0.0054,0.7038]$} & {$\left[1,-0.43^{\circ}, 45.27^{\circ}\right]$} & {$[-40,50,50.50]$} \\
6 & {$[0.0004,1.0000,0.0033]$} & {$\left[1,89.98^{\circ}, 89.81^{\circ}\right]$} & {$[0,100.54,0]$} \\
7 & {$[-0.0012,-1.0000,0.0007]$} & {$\left[1,-90.07^{\circ}, 89.96^{\circ}\right]$} & {$[0,-0.00,0]$} \\
8 & {$[1.0000,-0.0000,-0.0002]$} & {$\left[1,-0.00^{\circ}, 90.01^{\circ}\right]$} & {$[-0.00,0,0]$} \\
\hline \hline
\end{tabular}


TABLE V

RESULTS OF COMPARISON OF ALTERNATIVE ALGORITHMS IN CASE STUDY 2

\begin{tabular}{lclll}
\hline \hline Algorithm & $\begin{array}{c}\text { CPU } \\
\text { time [s] }\end{array}$ & Parameter & $\begin{array}{c}\text { Value of } \\
\text { parameter }\end{array}$ & $\begin{array}{c}\text { Detected plane } \\
{[\mathrm{r}, \theta, \varphi]^{\mathrm{a}}}\end{array}$ \\
\hline DWT based & 0.66 & Detail threshold: 0.025 & {$\left[97.50,89.93^{\circ}, 70.19^{\circ}\right]$} \\
3D Hough & 901.83 & Res. $(\theta, \varphi, \mathrm{r}):$ & $\begin{array}{l}5^{\circ}, 5^{\circ}, 0.5 \mathrm{~mm} \\
\text { Threshold: }\end{array}$ & $\begin{array}{l}0.2 \mathrm{~mm} \\
{\left[97.50,90^{\circ}, 70^{\circ}\right]}\end{array}$ \\
& & Range for $\mathrm{r}$ & $0 \div 135 \mathrm{~mm}$ & \\
& & No. of planes & 4 \\
RANSAC & 5.75 & Threshold & $0.2 \mathrm{~mm}$ & {$\left[97.50,89.94^{\circ}, 70.20^{\circ}\right]$} \\
& & No. of planes & 4 & \\
\hline \hline
\end{tabular}

${ }^{\mathrm{a}}$ An example of detected plane

part was made on a conventional milling machine. Fig. 9 presents the triangles on the points that were recognized as points belonging to the fitted planes. An example of plane recognition results obtained using alternative algorithms for surface visible from positive $\mathrm{z}$-axis is presented in Table V.

\section{Case Study 3 - Casting with Complex Geometry}

In the previous two examples we have used the test pieces whose geometry was known in advance. In this case study we will consider the piece shown in Fig. 10 - a casting that represents un-machined insert of an injection mold die whose geometry is unknown. This is a very interesting test piece since it has a number of cylindrical surfaces with relatively large diameters that can be easily mixed up with planar surfaces. In addition, the piece has a number of freeform surfaces. Besides, the flatness of planar surfaces obtained during casting process is extremely low as can be observed in Fig. 10a.

Using the proposed method six planar regions were recognized from point cloud. Three of them (visible from the selected viewpoint) are shown in Fig. 10b. The algorithm was
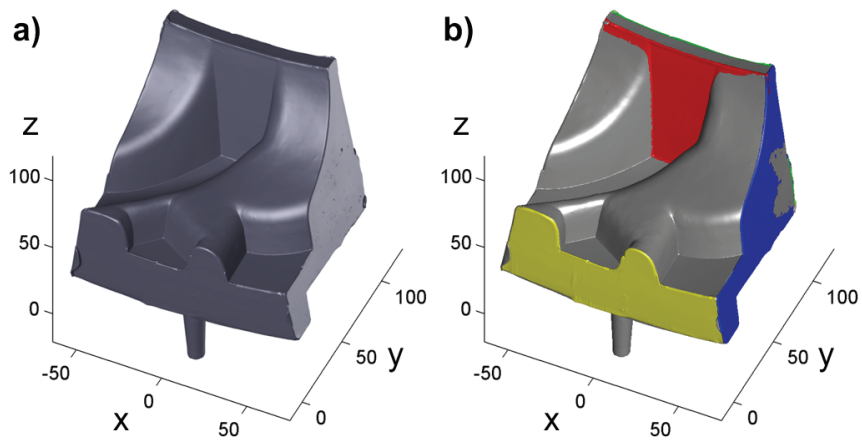

Fig. 10. Test piece in case study 3: a) Un-machined insert of an injection mold die; b) Detected planar regions in test piece from case study 3 .

TABLE VI

RESULTS OF COMPARISON OF ALTERNATIVE ALGORITHMS IN CASE STUDY 3

\begin{tabular}{|c|c|c|c|c|}
\hline Algorithm & $\begin{array}{c}\mathrm{CPU} \\
\text { time }[\mathrm{s}]\end{array}$ & Parameter & $\begin{array}{l}\text { Value of } \\
\text { parameter }\end{array}$ & $\begin{array}{c}\text { Detected plane } \\
{[\mathrm{r}, \theta, \varphi]^{\mathrm{a}}}\end{array}$ \\
\hline DWT based & 1.14 & Detail thresho & 0.0004 & {$\left[69.95,90.42^{\circ}, 100.1^{\circ}\right]$} \\
\hline 3D Hough & 3152.5 & $\begin{array}{l}\text { Res. }(\theta, \varphi, r) \text { : } \\
\text { Threshold: } \\
\text { Range for } r \\
\text { No. of planes }\end{array}$ & $\begin{array}{l}5^{\circ}, 5^{\circ}, 0.5 \mathrm{~mm} \\
0.05 \mathrm{~mm} \\
0 \div 171 \mathrm{~mm} \\
2\end{array}$ & {$\left[70,90^{\circ}, 100^{\circ}\right]$} \\
\hline RANSAC & 176.62 & $\begin{array}{l}\text { Threshold } \\
\text { No. of planes }\end{array}$ & $\begin{array}{l}0.05 \mathrm{~mm} \\
2\end{array}$ & {$\left[70.45,89.63^{\circ}, 99.76^{\circ}\right]$} \\
\hline
\end{tabular}

${ }^{\mathrm{a}}$ An example of detected plane able to adequately distinguish planar regions from cylindrical surfaces with large diameter, as well as from freeform surfaces. Furthermore, the errors in planar surfaces flatness were not recognized as belonging to detected regions, as can be observed from Fig. 10b.

In this case study we have opted to compare the performance of the selected three algorithms for surface visible from negative y direction. The results are presented in Table VI.

\section{Comparison of Alternative Recognition Methods}

For illustration of the performances of the developed method, we have compared its results to 3D Hough transform and RANSAC for all three case studies. Our discussion will consider the computation efficiency (i.e, required execution times), accuracy, and parameters of the considered algorithms.

\section{1) Computation Efficiency}

For real-time applications, one of the most important properties is the speed of the algorithm for recognition of planar segments from a point cloud (i.e., required computation time). In terms of speed, the proposed DWT based method has significantly outperformed both 3D Hough transform and RANSAC. When compared to DWT based algorithm and RANSAC, 3D Hough transform has shown especially low time efficiency. In our case studies, 3D Hough transform used 2-3 orders of magnitude more CPU time for recognition of planes (as can be seen in Tables III, V, and VI) than the proposed DWT based algorithm and RANSAC.

The computation times of RANSAC when compared to our algorithm were significantly higher -6.02 times in case study $1,8.71$ times in case study 2 , and 154.93 times in case study 3 . These differences in RANSAC's execution times in different case studies are caused by the randomness of initial points sampling. Namely, in case studies 1 and 2, a large number of points from the point clouds belong to planar segments and there was a higher probability that RANSAC would select initial points from planar regions in early iterations. On the other hand, the test piece in case study 3 contained a significant number of curved segments, resulting in the prolonged execution time. However, the DWT based method proposed in this paper has shown similar computational efficiency in all of the three case studies.

\section{2) Accuracy}

The second important property of the algorithms for plane detection is the accuracy of the estimated plane parameters. As can be observed from Tables III, V, and VI, plane parameters obtained by using DWT based method and RANSAC are similar since the estimation in these algorithms is data driven. The accuracy of 3D Hough transform, on the other hand, is determined by 3D Hough space quantization. In our experiments we have used a discretization step of $0.5 \mathrm{~mm}$ for $r$, and $5^{\circ}$ for $\theta$ and $\varphi$, and all of the estimated parameters were the multiplication product of these values. Fortunately, all the planes in these examples had normal vectors whose inclination angles to coordinate axes were close to multiplication product of $5^{\circ}$, so the results from $3 \mathrm{D}$ Hough transform are acceptable. 
TABLE VII

THE RANGE OF THRESHOLd PARAMETERS FOR SUCCESSFUl RECOGNITION OF Planes In CONSIDERED CASE STUDIES

\begin{tabular}{lccc}
\hline \hline & $\begin{array}{c}\text { DWT based } \\
\text { algorithm }\end{array}$ & $\begin{array}{c}\text { 3D Hough } \\
\text { transform [mm] }\end{array}$ & RANSAC [mm] \\
\hline Case study 1 & $0.0005-0.004$ & $0.02-0.4$ & $0.002-0.5$ \\
Case study 2 & $0.002-0.05$ & $0.15-0.4$ & $0.02-2.0$ \\
Case study 3 & $0.00035-0.0012$ & $0.007-0.4$ & $0.02-0.1$ \\
\hline \hline
\end{tabular}

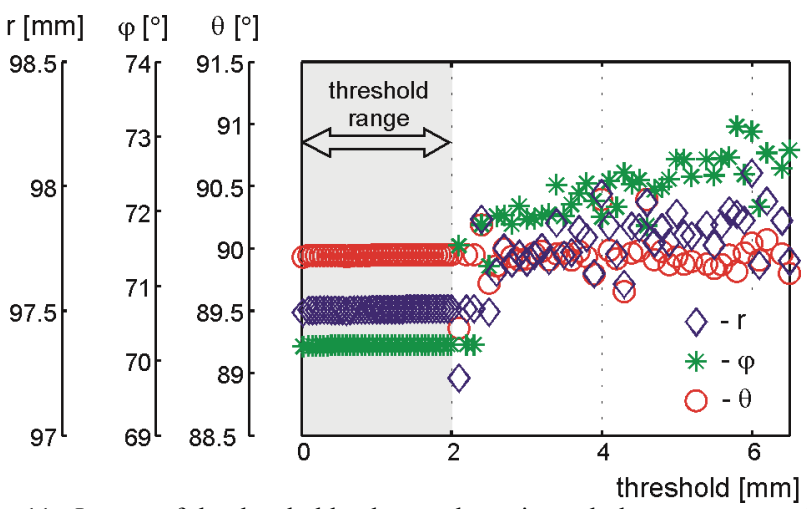

Fig. 11. Impact of the threshold value on the estimated plane parameters for RANSAC - plane from Table V, case study 2 .

However, if the planes with different normal vectors were present in point cloud, the 3D Hough transform with selected Hough space discretization would not provide acceptable accuracy. In this case, a higher resolution of Hough space would be required, introducing also a higher computation cost.

\section{3) Parameters of the Algorithms}

The third important property of the algorithms is the number of required parameters. The threshold is the parameter that is used in all three methods. In 3D Hough transform and RANSAC, the threshold represents the maximal distance between a recognized point on the plane and the estimated plane that point belongs to. On the other hand, in DWT based method the threshold can be experimentally obtained and depends on surface finish (i.e., roughness and waviness).

Successful recognition of planes from a point cloud demands appropriate assignment of the threshold value. Table VII presents acceptable threshold ranges for compared algorithms and considered case studies; the ranges were empirically obtained. For these threshold values, all planar segments were adequately recognized. The values of thresholds in ranges from Table VII do not have significant influence neither on the values of estimated plane parameters, nor on the computational efficiency. For example, in case study 2 , all the values of estimated parameters $r, \theta$, and $\varphi$, (for the plane from Table $\mathrm{V}$, and for different thresholds from Table VII) were in the range $\pm 0.005 \mathrm{~mm}, \pm 0.005^{\circ}$, and $\pm 0.005^{\circ}$ for DWT, and in the range $\pm 0.01 \mathrm{~mm}, \pm 0.01^{\circ}$, and $\pm 0.01^{\circ}$ for RANSAC. For Hough transform, as expected, the values of estimated plane parameters were exactly the same regardless the threshold value from Table VII. This illustrates that within the aforementioned threshold ranges, all three algorithms are quite robust to threshold variations.

Outside the threshold ranges from Table VII, the recognition results are as follows. If DWT based algorithm and Hough transform succeed to segment the plane from a point cloud, they will adequately estimate the plane parameters. On the other hand, for RANSAC, the accuracy of estimated plane parameters will depend on the selected threshold. Fig. 11 illustrates the effect of the threshold on the estimated values of $r, \theta$, and $\varphi$ for an example of the plane from Table V.

Another difference between methods is that when compared to DWT based algorithm and RANSAC, the number of parameters in 3D Hough transform is large. Besides threshold and 3D Hough space discretization steps, the range for $r$ should be provided. It is worth noting that to speed up the execution of 3D Hough transform and RANSAC, we have used an additional parameter - a number of planes to be recognized. Alternatively, a minimum number of detected points on a plane can be used to prevent the recognition of nonexistent planes.

\section{CONCLUSION}

In this paper, we have introduced a new method for segmentation of planar regions from a point cloud. The method is based on DWT and it exploits orthonormality of certain wavelets to linear and constant functions to distinguish planar segments from other surfaces in the cloud. Besides, the adjacent planar regions are segmented utilizing the asymmetry of wavelets and their sensitivity to abrupt changes.

The presented method employs a fast algorithm that is suitable for detection of multiple planar regions in the cloud. This algorithm is one pass, which is its advantage to RANSAC algorithm that demands a significant number of iterations in the presence of multiple planes. It is also not subject to the tradeoff between the velocity of execution and the accuracy of the plane parameters that characterizes Hough transform. In the presented method, the accuracy of plane parameters is data driven. The method does not require manual intervention typically present in region growing methods that are very sensitive to the seed selection. The only parameter of the proposed method is signal details threshold that depends on the roughness and flatness of the considered planar regions. In this paper, on three real-world case studies we have illustrated all the aforementioned advantages of the proposed algorithm over existing algorithms for planar segment detection.

The main drawback of the proposed method is that the algorithm requires a structured point cloud as input. To overcome this issue, we have used the derivative of $\mathrm{z}$ buffer algorithm for scattered point cloud structuring. However, it should be noted that a significant number of scanning devices outputs regular point sets, particularly as a low level output which is as a rule used in control tasks. In addition, although planar regions are crucial for a large number of 3D vision based industrial control tasks, the other types of surfaces (e.g., cylinders) are frequently met in manufacturing environments. The future work should address the segmentation of more complex surfaces (e.g., natural quadrics). 


\section{REFERENCES}

[1] S. Chen and Y.F. Li, "Finding Optimal Focusing Distance and Edge Blur Distribution for Weakly Calibrated 3-D Vision," IEEE Trans. Ind. Inf., vol. 9, no. 3, pp. 1680 - 1687, Aug. 2013.

[2] E. Savio, L. De Chiffr and R. Schmitt, "Metrology of freeform shaped parts," CIRP Ann.-Manuf. Techn., vol. 56, no. 2, pp. 810-834, 2007.

[3] Z. Fang, D. Xu, and M. Tan, "A Vision-Based Self-Tuning Fuzzy Controller for Fillet Weld Seam Tracking," IEEE/ASME Trans. Mechatronics, vol. 16, no. 3, pp 540 - 550, Jun. 2011.

[4] J. Xu, N. Xi, C. Zhang, Q. Shi, and J. Gregory, "A Robot-Assisted BackImaging Measurement System for Transparent Glass," IEEE/ASME Trans. Mechatronics, vol. 17, no. 4, pp. 779 - 788, Aug. 2012.

[5] Z. M. Bi, and L. Wang, "Advances in 3D data acquisition and processing for industrial applications," Robot. CIM-Int. Manuf., vol. 26, pp. 403-413, Oct. 2010.

[6] T. Varady, R. R. Martin, and J. Cox, "Reverse engineering of geometric models - an introduction," Comput. Aided Design, vol. 29, pp. 255-268, Apr. 1997.

[7] O. Schall, and M. Samozino, "Surface from Scattered Points: A Brief Survey of Recent Developments," in: Proc. 1st International Workshop towards Semantic Virtual Environments, Villars, Switzerland, 2005, pp. 138-147.

[8] W. B. Thompson, J. C Owen, H. J. de St. Germain, S. R. Jr Stark, and T.C. Henderson, "Feature-Based Reverse Engineering of Mechanical Parts," IEEE Trans. Robot. Autom., vol. 15, no. 1, pp. 57-66, Feb. 1999.

[9] H. ElMaraghy, G. Schuh, W. ElMaraghy, F. Piller, P. Schönsleben, M. Tseng, and A. Bernard, "Product variety management," CIRP Ann.Manuf. Techn., vol. 62, no. 2, pp 629-652, 2013.

[10] Z. Jakovljevic, and R. Puzovic, "Detection of planar segments in point clouds using wavelet transform," in: Proc. $35^{\text {th }}$ Int. Conf. on Production Engineering, Kopaonik, Serbia, 2013, pp. 205-208.

[11] S. Livatino, F. Banno and G. Muscato, "3-D Integration of Robot Vision and Laser Data With Semiautomatic Calibration in Augmented Reality Stereoscopic Visual Interface," IEEE Trans. Ind. Inf., vol. 8, no. 1, pp. $69-77$, Feb. 2012.

[12] J. Rodriguez-Araujo, J. J. Rodriguez-Andina, J. Farina, and M-Y. Chow, "Field-Programmable System-on-Chip for Localization of UGVs in an Indoor iSpace," IEEE Trans. Ind. Inf., vol. 10, no 2, pp. 1033 - 1043, May 2014.

[13] Y. Li, S. Li, Q. Song H. Liu and M.Q.-H. Meng, "Fast and Robust Data Association Using Posterior Based Approximate Joint Compatibility Test," IEEE Trans. Ind. Inf., vol. 10, no. 1, pp. 331 - 339, Feb. 2014.

[14] A. Corrales Paredes, M. Malfaz and M. A. Salichs, "Signage System for the Navigation of Autonomous Robots in Indoor Environments," IEEE Trans. Ind. Inf., vol. 10, no. 1, pp. 680 - 688, Feb. 2014.

[15] G. Fu, P. Corradi, A. Menciassi, and P. Dario, "An Integrated Triangulation Laser Scanner for Obstacle Detection of Miniature Mobile Robots in Indoor Environment," IEEE/ASME Trans. Mechatronics, vol. 16, no. 4, pp. 778 - 783, Aug. 2011.

[16] B. Mobedi, and G. Nejat, "3-D Active Sensing in Time-Critical Urban Search and Rescue Missions," IEEE/ASME Trans. Mechatronics, vol. 17, no. 6, pp 1111 - 1119, Dec. 2012.

[17] D. Borrmann, J. Elseberg, K. Lingemann, and A. Nuechter, "The 3D Hough transform for plane detection in point clouds: a review and a new accumulator design," 3DR Expresss, vol. 2, art. 32, Nov. 2011.

[18] B. Oehler, J. Stueckler, J. Welle, D. Schulz, and S. Behnke, "Efficient Multi-Resolution Plane Segmentation of 3D Point Clouds," in: Proc. of the 4th Int. Conf. on Intelligent Robotics and Applications, vol. II, Aachen, Germany, 2011, pp. 145-156.

[19] J. Li and N. Allinson, "Building Recognition Using Local Oriented Features," IEEE Trans. Ind. Inf., vol. 9, no. 3, pp. 1697 - 1704, Aug. 2013.

[20] F. Tarsha-Kurdi, T. Landes, and P. Grussenmeyer, "Hough transform and extended RANSAC algorithms for automatic detection of $3 \mathrm{~d}$ building roof planes from lidar data," in: Proc. ISPRS Workshop Laser Scanning 2007 and SilviLaser 2007, Finland, vol. 36, pp. 407-412.

[21] K. Schindler, and J. Bauer, "A model-based method for building reconstruction," in: Proc. First IEEE Int. Workshop on Higher-Level Knowledge in 3D Modeling and Motion Analysis, Nice, France, 2003, pp. $74-82$.

[22] D. U. Lee, L. W. Kim and J. D. Villasenor, "Precision-Aware SelfQuantizing Hardware Architectures for the Discrete Wavelet Transform," IEEE Trans. Image Process., vol. 21, no. 2, pp. 768-777, Feb. 2012.
[23] F. Aulí-Llinàs, General Embedded Quantization for Wavelet-Based Lossy Image Coding, IEEE Trans. Signal Process., vol. 61, no. 6, pp. 1561-1574, Mar. 2013

[24] R. Yan, L. Shao and Y. Liu, "Nonlocal Hierarchical Dictionary Learning Using Wavelets for Image Denoising," IEEE Trans. Image Process., vol. 22, no. 12, pp. 4689-4698, Dec. 2013.

[25] S. G. Roux, M. Clausel, B. Vedel, S. Jaffard and P. Abry, "Self-Similar Anisotropic Texture Analysis: The Hyperbolic Wavelet Transform Contribution," IEEE Trans. Image Process., vol. 22, no. 11, pp. $4353-$ 4363, Nov. 2013.

[26] A. Othmani, L. F.C. Lew Yan Voon, C. Stolz and A. Piboule, "Single tree species classification from Terrestrial Laser Scanning data for forest inventory," Pattern Recogn. Lett., vol. 34, no. 16, pp. 2144-2150, Dec. 2013.

[27] D. Castaño and A. Kunoth, "Multilevel regularization of wavelet based fitting of scattered data - some experiments," Numer. Algorithms, vol. 39, no. 1-3, pp. 81-96, Jul. 2005.

[28] G. Xu, X. Cheng, L. Wang, K. Tan and Q. Lou, "An Adaptive Compression Algorithm of Scattered Point Cloud Based on Wavelet Technology," J. Inf. \& Comput. Sci., vol. 11, no. 6, pp. 1917-1927, Apr. 2014

[29] M. Kedzierski and A. Fryskowska, Terrestrial and Aerial Laser Scanning Data Integration Using Wavelet Analysis for the Purpose of 3D Building Modeling, Sensors-Basel, vol. 14, no. 7, pp. 12070-12092, Jul. 2014.

[30] Method and Means for Recognizing Complex Patterns, by P. V. C. Hough, (1962). U.S. Patent 3.069.654.

[31] M. A. Fischler, and R. C. Bolles, "Random Sample Consensus: A paradigm for model fitting with application to image analysis and automated cartography," Commun. ACM, vol. 24, pp. 381-395, Jun. 1981

[32] R. Schnabel, R. Wahl, and R. Klein, "Efficient RANSAC for PointCloud Shape Detection," Comput. Graph. Forum, vol. 26, no. 2 pp. 214226, Jun. 2007.

[33] D. Cohen-Steiner, P. Alliez, and M. Desbrun, "Variational Shape Approximation," ACM T. Graphic., vol. 23, no. 3, pp 905-9014, Aug. 2004

[34] M. Garland, A. J. Willmott, and P. S. Heckbert, "Hierarchical face clustering on polygonal surfaces," in: Proc. 2001 Symp. on Interactive 3 graphics, New York, 2001, pp. 49-58.

[35] M. Attene, B. Falcidieno, and M. Spagnuolo, "Hierarchical mesh segmentation based on fitting primitives," Visual Comput., vol. 22, pp. 181-193, Mar. 2006.

[36] J. Poppinga, N. Vaskevicius, A. Birk, and K. Pathak, "Fast Plane detection and polygona-lization in noisy 3D images," in Proc. Int. Conf on Intelligent Robots and Systems, Nice France, 2008, pp. 3378 - 3383.

[37] R. Lakaemper, and L. J. Latecki, "Extended EM for Planar Approximation of 3D Data," in Proc. IEEE Int. Conf. Robot., Orlando, Florida, 2006, pp. $1173-1179$.

[38] L. Wang, J. Cao, and C. Han, "Multidimensional particle swarm optimization-based unsupervised planar segmentation algorithm of unorganized point clouds," Pattern Recogn., vol. 45, no. 11, pp 40344043, Nov. 2012.

[39] H. H. Nguyen, J. Kim, Y. Lee, N. Ahmed, and S. Lee, "Accurate and fast extraction of planar surface patches from 3D point cloud," in: Proc. 7 th Int. Conf. on Ubiquitous Information Management and Communication, 2013, Article No. 84.

[40] J. Xiao, J. Zhang, B. Adler, H. Zhang and J. Zhang, "Three-dimensional point cloud plane segmentation in both structured and unstructured environments," Robot. Auton. Syst., vol. 61, pp.1641 - 1652, Dec. 2013.

[41] I. Daubechies, Ten Lectures on Wavelets. CBMS-NSF regional conference series in applied mathematics, Vol. 61, SIAM, 1992.

[42] S. G. Mallat, "A Theory for Multiresolution Signal Decomposition: The Wavelet Representation," IEEE Trans Pattern Anal and Mach Intell, vol. 11, no.7, pp. 674-693, Jul. 1989.

[43] A. Antoniadis, J. Bigot, T. Sapatinas, "Wavelet estimators in nonparametric regression: A comparative simulation study," J. Stat. Soft., vol. 6, pp. 1-83, Jun. 2001.

[44] L. G. Shapiro, "Connected Component Labeling and Adjacency Graph Construction," in Topological Algorithms for Digital Image Processing, Machine Intelligence and Pattern Recognition, vol. 19, T. Y. Kong and A. Rosenfeld, Ed. Amsterdam, Netherlands: Elsevier, 1996, pp. 1-30.

[45] P. Benkő, R. R. Martin, and T. Várady, "Algorithms for reverse engineering boundary representation models," Comput. Aided Design, vol. 33, pp. 839-851, Sep. 2001 
[46] R, Sacchi, J. F. Poliakoff, P. D. Thomas, and K. -H Hafele, "Improved extraction of planar segments for scanned surfaces," in: Proc. IEEE Int. Conf Inf. Vi., London, 2000, pp. $325-330$.

[47] S. Petitjean, "A survey of methods for recovering quadrics in triangle meshes," ACM Comput. Surv., vol. 34, no. 2, pp. 211-262, Jun. 2002.

[48] J. A. P. Kjellander, and M. Rahayem, "Planar segmentation of data from a laser profile scanner mounted on an industrial robot," Int. J. Adv. Manuf. Tech., vol. 45, no 1-2, pp. 181-190, Nov. 2009.

[49] GOM GmbH, "ATOS Compact Scan - The compact class of scanning," 2014 [Online]. Available: http://www.gom.com/metrologysystems/system-overview/atos-compact-scan.html.

[50] E. Catmull, "A Subdivision Algorithm for Computer Display of Curved Surfaces," Ph.D. Dissertation, Dept. Comput. Sci., Univ. of Utah, Salt Lake City, UT, 1974.

[51] P. D. Kovesi. (2014, Sept.). MATLAB and Octave Functions for Computer Vision and Image Processing. Centre for Exploration Targeting, School of Earth and Environment, The University of Western Australia. Perth. Australia. [Online]. Available: http://www.csse.uwa.edu.au/ pk/research/matlabfns/>.

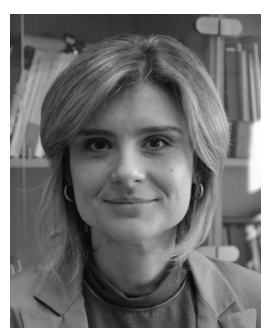

Zivana Jakovljevic received Dipl. Ing., M.Sc. and $\mathrm{Ph} . \mathrm{D}$. degrees in mechanical engineering from the Faculty of Mechanical Engineering, University of Belgrade, Serbia, in 1999, 2004 and 2010, respectively.

Since 2001 she is the member of academic staff at the Faculty of Mechanical Engineering, University of Belgrade, Serbia, first as a Research and Teaching Assistant, and from 2011 as Assistant Professor. Her research interests include intelligent manufacturing systems and in particular mathematical pattern recognition based on soft computing, and non-stationary signal processing and its application in manufacturing automation and computer graphics.

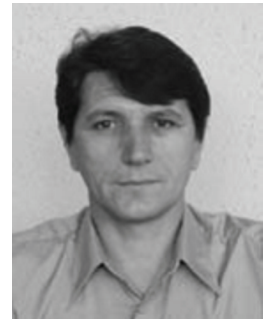

Radovan Puzovic received Dipl. Ing., M.Sc. and $\mathrm{Ph} . \mathrm{D}$. degrees in mechanical engineering from the Faculty of Mechanical Engineering, University of Belgrade, Serbia, in 1987, 1993 and 2000, respectively. He is currently an Associate Professor at the Department for Production Engineering, Faculty of Mechanical Engineering, University of Belgrade, Serbia. His research interests include $\mathrm{CAD} / \mathrm{CAM}$ systems, manufacturing technology, and machining processes.

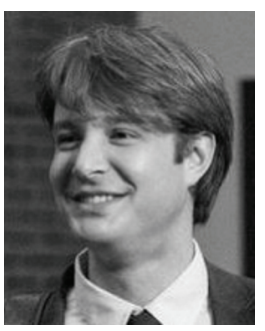

Miroslav Pajic (S'06-M'14) received the Dipl. Ing. and M.S. degrees in electrical engineering from the University of Belgrade, Serbia, in 2003 and 2007, respectively, and the M.S. and Ph.D. degrees in electrical engineering from the University of Pennsylvania, Philadelphia, in 2010 and 2012, respectively.

$\mathrm{He}$ is currently an Assistant Professor in the Department of Electrical and Computer Engineering at Duke University. His research interests focus on the design and analysis of cyber-physical systems and in particular real-time and embedded systems, distributed/networked control systems, advanced manufacturing and high-confidence medical device systems.

Dr. Pajic has received various awards including the 2011 ACM SIGBED Frank Anger Memorial Award, the Joseph and Rosaline Wolf Award for Best Dissertation from Penn Engineering, the Best Paper Award at the 2014 ACM/IEEE International Conference on Cyber-Physical Systems (ICCPS), and the Best Student Paper award at the 2012 IEEE Real-Time and Embedded Technology and Applications Symposium (RTAS). 